\title{
THREE-DIMENSIONAL NANOCOMPOSITE SCAFFOLDS FOR BONE TISSUE ENGINEERING: FROM DESIGN TO APPLICATION
}

\author{
Bin Duan ${ }^{1, *}$, Min Wang ${ }^{1, *}$, William W. Lu ${ }^{2}$ \\ ${ }^{1}$ Department of Mechanical Engineering, Faculty of Engineering \\ The University of Hong Kong, Pokfulam Road, Hong Kong \\ *memwang@hku.hk \\ ${ }^{2}$ Department of Orthopaedics \& Traumatology, Li Ka Shing Faculty of Medicine \\ The University of Hong Kong, Sassoon Road, Hong Kong \\ * Current address: Department of Biomedical Engineering, Cornell University \\ Ithaca, NY 14853-7202, USA
}

\author{
Received Day Month Year \\ Revised Day Month Year
}

\begin{abstract}
Selective laser sintering (SLS), a rapid prototyping technology, was investigated for producing bone tissue engineering scaffolds. Totally biodegradable, osteoconductive calcium phosphate (CaP)/poly(hydroxybutyrate-co-hydroxyvalerate) (PHBV) scaffolds were successfully fabricated via SLS using Ca-P/PHBV nanocomposite microspheres. In the SLS manufacturing route, the architecture of tissue engineering scaffolds (pore shape, size, interconnectivity, etc.) can be designed and the sintering process can be optimized for obtaining scaffolds with desirable porous structures and mechanical properties. SLS was also shown to be very effective in producing highly complex porous structures using nanocomposite microspheres. To render SLS-formed Ca-P/PHBV scaffolds osteoinductive, recombinant human bone morphogenetic protein-2 (rhBMP-2) could be loaded onto the scaffolds. For achieving a controlled release of rhBMP-2 from scaffolds, surface modification of Ca-P/PHBV scaffolds by gelatin entrapment and heparin immobilization was needed. The immobilized heparin provided binding affinity for rhBMP-2. Surface modified Ca-P/PHBV nanocomposite scaffolds loaded with rhBMP-2 enhanced the proliferation of human umbilical cord derived mesenchymal stem cells (hUCMSCs) and also their alkaline phosphatase activity. In in vivo experiments using a rabbit model, surface modified Ca-P/PHBV nanocomposite scaffolds loaded with rhBMP-2 promoted ectopic bone formation, exhibiting their osteoinductivity. The strategy of combining advanced scaffold fabrication, nanocomposite material and controlled growth factor delivery is promising for bone tissue regeneration.
\end{abstract}

Keywords: bone tissue engineering, scaffold, selective laser sintering, nanocomposite, growth factor, controlled release.

\section{Introduction}

Tissue engineering emerged two decades ago, holding the promises of providing long-term solutions in human body tissue repair and potentially offering treatments for medical conditions that are currently untreatable [1]. For bone tissue repair, combining biodegradable materials (in the form of porous scaffolds) with living cells and/or bioactive component(s) for bone regeneration provides an alternative to autologous bone grafts, the gold standard in current orthopaedic surgery, and allogenous bone grafts [2]. In scaffold-based bone tissue engineering, scaffolds play an important role by mimicking the 
structure and functions of the extracellular matrix (ECM) of the tissue and by providing an interconnected porous structure for new bone formation and vascularization [3]. Bone is a natural nanocomposite material and therefore nanocomposites consisting of a biodegradable polymer matrix and nanosized osteoconductive fillers such as bioceramics and bioglasses can be used to construct bone tissue engineering scaffolds [4], enhancing bone tissue formation. Furthermore, controlled growth factor delivery using the scaffolds as delivery vehicles can be employed to promote bone regeneration. Owing to their short half-lives, rapid degradation rate, relatively large size and slow tissue penetration, direct therapeutic applications of growth factors have been limited [5]. Using scaffolds for the controlled delivery of growth factors can overcome the problems. The generally adopted strategies for incorporating growth factors into scaffolds include encapsulation in the scaffold, physical adsorption and chemical immobilization on pore surfaces of scaffolds [6,7].

Rapid prototyping (RP) techniques are matured manufacturing technologies which have been widely used in traditional industries during the design process [8]. RP comprises a group of technologies that can generate a physical model directly from computeraided design data in a layer-by-layer manner and each layer is in the shape of the cross-section of the model at a specific level [9]. From the traditional industries, RP techniques were first borrowed and employed for medical applications when they were used to produce accurate models or prosthesis for surgical planning and simulation [10, 11]. With the emergence and development of tissue engineering, various biomaterials and RP techniques are being investigated to specifically fabricate porous scaffolds with controlled architectures (pore size, shape, interconnectivity, geometry and orientation, etc.) and properties, targeting at the regeneration of different tissues [12-15]. The advantages of RP techniques over conventional scaffold fabrication methods include, but not limited to, achieving well defined external and internal architectures of the scaffolds, computer controlled manufacturing processes, and high accuracy and reproducibility. Therefore, more and more RP techniques are now investigated, developed and commercialized for tissue engineering scaffold fabrication utilizing biocompatible and biodegradable materials as raw materials.
In the current investigation, selective laser sintering (SLS), which is an established RP technology, was employed to fabricate three-dimensional (3D) nanocomposite scaffolds using calcium phosphate (Ca$\mathrm{P}) /$ poly(hydroxybutyrate-co-hydroxyvalerate) (PHBV) nanocomposite microspheres. The surface of $\mathrm{Ca}$ P/PHBV nanocomposite scaffolds was modified through physical entrapment of gelatin and then immobilization of heparin in order to provide growth factor binding sites. The surface modified scaffolds were subsequently loaded with recombinant human bone morphogenetic proteins-2 (rhBMP-2). The effects of surface modification and rhBMP-2 on osteogenic differentiation of human umbilical cord derived mesenchymal stem cells (hUCMSCs) were studied. Bone tissue regeneration using rhBMP-2 loaded nanocomposites was investigated using a rabbit model.

\section{Materials and Methods}

\subsection{Fabrication of Ca-P/PHBV nanocomposite microspheres}

SLS requires powdered raw materials with appropriate particle sizes. Therefore, Ca-P/PHBV nanocomposite microspheres consisting of $\mathrm{Ca}-\mathrm{P}$ nanoparticles and PHBV matrix were firstly fabricated. As shown in Figure 1A, Ca-P nanoparticles were synthesized inhouse by rapid mixing of $\mathrm{Ca}\left(\mathrm{NO}_{3}\right)_{2} \cdot 4 \mathrm{H}_{2} \mathrm{O}$ acetone solution with aqueous $\left(\mathrm{NH}_{4}\right)_{2} \mathrm{HPO}_{4}$ solution [16]. With sizes in the range of $10-30 \mathrm{~nm}$, the Ca-P nanoparticles synthesized were amorphous and had a Ca:P molar ratio of 1.49. PHBV with $12 \mathrm{~mol} \%$ 3-hydroxyvalerate was from ICI, UK. As shown in Figure 1B, the Ca$\mathrm{P} / \mathrm{PHBV}$ nanocomposite microspheres were prepared using a solid-in-oil-in-water $(\mathrm{S} / \mathrm{O} / \mathrm{W})$ emulsion solvent evaporation method [17]. Their Ca-P content was 12.9 $\mathrm{wt} \%$ (targeting at $15 \mathrm{wt} \%$ ), as was determined through thermal gravimetric analysis (TGA).

\subsection{Design and fabrication of $3 D$ nanocomposite scaffolds}

A modified Sinterstation ${ }^{\circledR 2000}$ system (3D Systems, Valencia, CA, USA), as shown in Figure 1C, was used for selective laser sintering of scaffolds or porous structures. The modification of the SLS machine for 


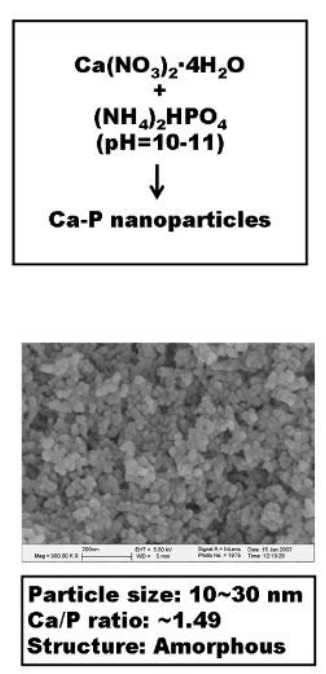

A
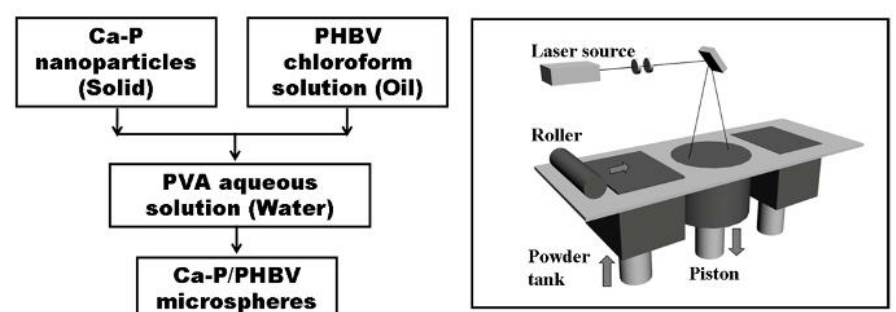

microspheres

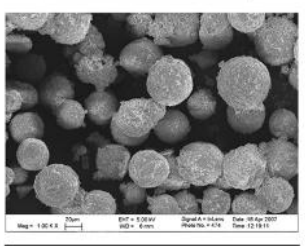

Particle size: $\mathbf{4 6 . 3 4} \mu \mathrm{m}$ Ca-P content: $\sim 12.9 \%$

B

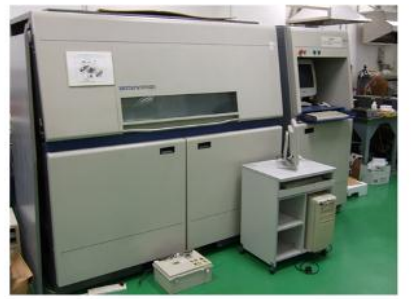

C

Fig. 1. Manufacturing process for Ca-P/PHBV nanocomposite scaffolds: (A) synthesis of Ca-P nanoparticles; (B) fabrication of Ca-P/PHBV nanocomposite microspheres using the $\mathrm{S} / \mathrm{O} / \mathrm{W}$ emulsion solvent evaporation method; (C) principle of SLS and a modified commercial Sinterstation ${ }^{\circledR} 2000$ SLS system for scaffold fabrication.

scaffold fabrication was reported previously [18]. In order to assess the capability of SLS in constructing porous structures/scaffolds of complex shapes and architecture, four complex models (Figure 2A) designed by Hart [19] were employed for producing porous structures via SLS. Simple porous scaffold models with 3D periodic architectures (e.g., Figure 3A, Figure 4A and Figure 7A) were designed using SolidWorks ${ }^{\circledR}$ for different purposes. For optimizing pore size, three scaffold models with similar scaffold architecture but different pore sizes, as shown in Figure $3 \mathrm{~A}$, were designed and their dimensions are listed in Table 1.

Table 1 Design parameters of three scaffold models for selective laser sintering

\begin{tabular}{ccccc}
\hline Type & $\begin{array}{c}\text { Scaffold Size } \\
\left(\mathrm{mm}^{3}\right) *\end{array}$ & $\begin{array}{c}\text { Pore Size } \\
\left(\mathrm{mm}^{3}\right)\end{array}$ & $\begin{array}{c}\text { Strut } \\
\text { Size } \\
(\mathrm{mm})\end{array}$ & $\begin{array}{c}\text { Porosity } \\
(\%) * *\end{array}$ \\
\hline 1 & $8.0 \times 8.0 \times 15.5$ & $1.0 \times 1.0 \times 1.0$ & 0.5 & 67.9 \\
2 & $8.3 \times 8.3 \times 13.5$ & $0.8 \times 0.8 \times 0.8$ & 0.5 & 52.7 \\
3 & $8.2 \times 8.2 \times 11.5$ & $0.6 \times 0.6 \times 0.6$ & 0.5 & 38.0
\end{tabular}

\footnotetext{
* Excluding the base.

** Theoretical porosity according to scaffold design.
}

The designs were exported into an STL format and transferred to the SLS machine for scaffold production. According to our previous optimization results, the laser power was set at $15 \mathrm{~W}$ and the scan spacing and layer thickness were $0.1 \mathrm{~mm}$ and $0.1 \mathrm{~mm}$, respectively. The part bed temperature, scan speed and roller speed were $35{ }^{\circ} \mathrm{C}, 1257 \mathrm{~mm} / \mathrm{s}$, and $127 \mathrm{~mm} / \mathrm{s}$, respectively.

\subsection{Scaffold surface modification}

The surface modification of rod-shaped sintered scaffolds was conducted in two steps: (1) physical entrapment of gelatin, and (2) heparin immobilization. The physical entrapment of gelatin was achieved by immersing sintered Ca-P/PHBV scaffolds (two layers, $\sim 70 \mathrm{mg} /$ each scaffold) into a gelatin (Sigma, USA) solution, which used a miscible mixture of 2,2,2trifluoroethanol (TFE; Acros, Belgium) and distilled water (TFE:water=30:70), at room temperature for 6 hr. After the treatment, the scaffolds were rinsed in distilled water three times to remove non-entrapped gelatin and the dried at room temperature. The amount of gelatin entrapped on the scaffold surface was determined by bicinchoninic acid (BCA; Pierce, Rockford, IL, USA) kit assay. For heparin immobilization, gelatin-entrapped Ca-P/PHBV 
scaffolds were first prewetted in 2-(Nmorpholino)ethanesulfonic acid (MES) buffer solution (0.1 M, pH 5.6; Fluka, USA) for $30 \mathrm{~min}$ at room temperature. $3 \mathrm{mg}$ of heparin $(\mathrm{Mw}=17,000$, activity 170USP units/mg; Sigma, USA) were dissolved in 1 $\mathrm{ml}$ MES buffer solution (0.1 M, pH5.6) containing 2 mg N-hydroxysuccinimide (NHS; Sigma, USA) and $1.2 \mathrm{mg}$ 1-ethyl-3-(3-dimethylaminopropyl)carbodiimide hydrochloride (EDC; Sigma, USA). After $4 \mathrm{hr}$ activation at room temperature, the prewetted scaffolds were soaked in the activated heparin solution in MES buffer for another $4 \mathrm{hr}$ and then extensively washed with phosphate buffered saline (PBS) and dried overnight at room temperature. The amount of heparin conjugated to the surface of gelatin entrapped scaffold was determined using the toluidine blue method.

\subsection{Characterization}

The morphology of sintered scaffolds was examined using scanning electron microscopes (LEO $1530 \mathrm{FE}$ SEM or Hitachi S-3400N SEM) and the porosity of scaffolds was measured using a density kit and an electronic balance on the basis of Archimedes principle. For mechanical testing, sintered, fourlayered scaffold samples were used. Compression tests were performed using an Instron 5848 MicroTester and the crosshead speed was set at $1.0 \mathrm{~mm} / \mathrm{min}$.

Due to the high porosity of scaffolds designed and sintered, instead of using sintered scaffolds, the hydrophilicity of scaffold surface before and after surface modification was studied by measuring contact angles of distilled water droplets on PHBV films which were produced using the solvent-casting method. The surface modification of solvent-cast PHBV films adopted the same procedure as described in previous sections. A contact angle machine (SL200B, Solon Tech, Co., Ltd., Shanghai, China) equipped with an image analyzing system was used and water contact angles were measured at room temperature using the sessile drop technique.

\subsection{In vitro biological study}

\subsubsection{Culture of hUCMSCs}

hUCMSCs were provided by Allianceslls Bioscience Co., Beijing, China. In cell culture, after reaching $80 \%$ confluence, cells were digested and subcultured using $0.25 \%(\mathrm{w} / \mathrm{v})$ trypsin-ethylenediamineteraacetic acid (EDTA) (Invitrogen, USA). The resulting cells in a suspension were seeded separately onto two-layered scaffolds which had been sterilized by ${ }^{60} \mathrm{Co} \gamma$ irradiation. For control experiments, polystyrene tissue culture plates (TCPs) were used. Cell seeding onto samples was conducted by dripping $100 \mu$ l cell suspension $\left(5 \times 10^{6}\right.$ cells $\left./ \mathrm{ml}\right)$ in 24 -well plates and the culture wells were filled with $1 \mathrm{ml}$ of culture medium after $2 \mathrm{hr}$ in the incubator.

\subsubsection{Cell morphology}

After 21 day culture in an osteogenic medium (the basal medium supplemented with $10 \mathrm{nM}$ dexamethasone, $0.05 \mathrm{mM}$ L-ascorbic acid, and $0.01 \mathrm{mM} \beta$-glycerophosphate.), cell-scaffold constructs were harvested, washed with PBS and fixed with $2.5 \%$ glutaraldehyde at $4{ }^{\circ} \mathrm{C}$ for $4 \mathrm{hr}$. After washing with cacodylate buffer containing $0.1 \mathrm{M}$ sucrose, they were dehydrated through a series of graded alcohol solutions and dried in a critical point dryer using liquid carbon dioxide as the transition fluid. The samples were coated with a thin layer of gold before SEM examination.

\subsubsection{Cell proliferation}

After cell culture of 1, 4 and 7 days in a basal medium (DMEM/F12), the proliferation of hUCMSCs on CaP/PHBV scaffolds with and without surface modification were determined using 3-(4,5dimethylthiazol-2-yl)-2,5-diphenyltetrazolium bromide (MTT) assay. At these time points, the culture medium was removed and cell-scaffold constructs were washed with PBS to remove non-adhering cells. Culture medium with MTT solution $(5 \mathrm{mg} / \mathrm{ml}$ in PBS) was added to each cell-scaffold sample, followed by incubation for $4 \mathrm{hr}$ at $37{ }^{\circ} \mathrm{C}$. After removal of the medium, the formazan crystals formed were dissolved in dimethyl sulfoxide (DMSO) and the absorbance was 
measured using a spectrophotometer at the wavelength of $570 \mathrm{~nm}$.

\subsubsection{Osteogenic differentiation}

For studying the osteogenic differentiation of hUCMSCs on the scaffolds, four groups of scaffold samples were used: Ca-P/PHBV scaffolds, CaP/PHBV scaffolds with rhBMP-2, Ca-P/PHBV scaffolds with surface modification, and surface modified Ca-P/PHBV scaffolds with rhBMP-2. For incorporating rhBMP-2, $5 \mu \mathrm{g}$ rhBMP-2 were loaded onto each scaffold with and without surface modification by dripping $50 \mu \mathrm{l}$ rhBMP-2 solutions with $20 \mathrm{mM}$ glacial acetic acid and $0.1 \%$ bovine serum albumin (BSA).

\subsubsection{Alkaline phosphatase (ALP) activity}

The ALP activity was measured after 7, 14 and 21 day cell culture after osteogenic induction. Cell-scaffold constructs were rinsed with PBS to remove nonadhering cells. They were treated with $200 \mu$ trypsin solution to detach cells. $500 \mu \mathrm{l}$ culture medium were then added to end the digestion. After centrifugation at $5000 \mathrm{rpm}$ for $5 \mathrm{~min}$ and further wash with PBS, the cells obtained were kept in $500 \mu \mathrm{l}$ lysis buffer containing $0.1 \%(\mathrm{v} / \mathrm{v})$ Triton $\mathrm{X}-100,1 \mathrm{mM} \mathrm{MgCl}$, and $20 \mathrm{mM}$ Tris, followed by a freezing and thawing process to further disrupt the cell membrane. $50 \mu \mathrm{l}$ lysate were mixed with $200 \mu \mathrm{l}$ ALP substrate solution containing p-nitrophenyl phosphate (pNPP) (Sigma, USA) at $37^{\circ} \mathrm{C}$ for $30 \mathrm{~min}$. The reaction was stopped by the addition of $50 \mu \mathrm{l}$ of $3 \mathrm{~N} \mathrm{NaOH}$. The production of p-nitrophenol in the presence of ALPase was then measured by monitoring the solution absorbance at $405 \mathrm{~nm}$ using a microplate reader. The total protein content was determined using BCA assay kit and the ALP activity was expressed as $\mu \mathrm{mol}$ of p-nitrophenol formation per minute per milligram of total proteins ( $\mu \mathrm{mol} / \mathrm{min} / \mathrm{mg})$.

\subsection{In vivo study for ectopic bone formation using a rabbit model}

\subsubsection{Animal and surgical procedure}

The in vivo study of Ca-P/PHBV scaffolds with or without rhBMP-2 was performed using a rabbit model.
All treatments and surgical procedures complied with the Regulation of Laboratory Animal Unit of the University of Hong Kong. Mature male New Zealand white rabbits weighing $3.5 \mathrm{~kg}$ to $4.0 \mathrm{~kg}$ were used. Prior to surgery, the rabbits were anaesthetized by intramuscular injection of a combination of ketamine $(35 \mathrm{mg} / \mathrm{kg})$, xylazine $(5 \mathrm{mg} / \mathrm{kg})$ and acepromzine $(1$ $\mathrm{mg} / \mathrm{kg})$. One dose of cephalexin $(15 \mathrm{mg} / \mathrm{kg})$ was also subcutaneously injected as an antibiotic before operation. The surgical site was shaved and disinfected with betadine and draped well by sterilized towel. A muscle-pocket in the left side along the posterior superior iliac spine and the lateral cortex of the ilium was carefully prepared, The surface modified scaffold loaded with rhBMP-2 was inserted in this pocket and then fully wrapped by muscle tissue (Figure 7B). The procedure was repeated on the right side of the rabbit, inserting the scaffold without surface modification and rhBMP-2 in the muscle-pocket. After operation, the general health and weight-bearing status of rabbits were monitored during recovery. The animals were sacrificed after 6 weeks and 12 weeks of surgery, respectively, by intravenous injection of a lethal dose of pentobarbital, and the scaffolds with surrounding tissues were explanted for subsequent experiments.

\subsubsection{Histological evaluation}

All samples harvested were fixed in 10\% buffered formalin for 3 days and then rinsed in running water for $2 \mathrm{hr}$. After further dehydration in a graded series of ethanol and subsequent immersion in xylene, the samples were embedded in polymethylmethacrylate (PMMA). The embedded samples were cut into sections with a thickness of about 200-300 $\mu \mathrm{m}$ by an EXAKT 300 CP Band System (Norderstedt, Germany) and micro-grounded down to 50-70 $\mu \mathrm{m}$ thick using an EXAKT 400 CP Micro Grinding System (Norderstedt, Germany). The thin samples were stained with giemsa-eosin and viewed under a light microscope (Nikon H600L, Japan).

\subsection{Statistical analysis}

All data were obtained from triplicate samples and expressed as the mean \pm standard deviation (SD). Statistical analysis was performed using ANOVA with a Scheffé test. A value of $p<0.05$ was considered to be 
statistically significant and $p<0.01$ remarkably significant.

\section{Results}

\subsection{Design and fabrication of scaffolds}

SLS employs a $\mathrm{CO}_{2}$ laser to selectively sinter thin layers of powdered polymers or their composites and form solid 3D objects. Therefore, Ca-P/PHBV nanocomposite microspheres, which served as the raw material for scaffold construction via SLS, were first fabricated. With sizes of 10-30 nm, the Ca-P nanoparticles synthesized (Figure 1A) were amorphous and had a Ca:P molar ratio of 1.49. Ca$\mathrm{P} / \mathrm{PHBV}$ nanocomposite microspheres based on Ca-P nanoparticles and biodegradable PHBV were produced using the $\mathrm{S} / \mathrm{O} / \mathrm{W}$ emulsion-solvent evaporation method (Figure 1B). In previous investigations, the distribution of Ca-P nanoparticles inside nanocomposite microspheres was studied [16]. Nanocomposite microspheres were embedded in an epoxy resin and then sectioned using a microtome. The cross-sectional view of Ca-P/PHBV nanocomposite microspheres were obtained through SEM and the distributions of calcium and phosphorus in the crosssections were conducted using energy dispersive X-ray spectroscopy (EDX). It was observed that calcium and phosphorus elements were homogeneously distributed in the cross-section, indicating the homogeneous encapsulation of $\mathrm{Ca}-\mathrm{P}$ nanoparticles in nanocomposite microspheres. Transmission electron microscopy (TEM) was also used to study the morphology and microstructure of $\mathrm{Ca}-\mathrm{P} / \mathrm{PHBV}$ nanocomposite microspheres. Apart from the encapsulation of Ca-P nanoparticles inside the microspheres, some agglomerated Ca-P nanoparticles were embedded on the microsphere surface. In the current investigation, PHBV microspheres were also made. Both PHBV and $\mathrm{Ca}-\mathrm{P} / \mathrm{PHBV}$ microspheres were of suitable sizes for the SLS technology. For sintering Ca-P/PHBV nanocomposite scaffolds, a miniature sintering platform containing two small powder supply tanks and one small build cylinder tank was designed and installed in the build cylinder of existing Sinterstation ${ }^{\circledR 2000}$ system [18], as shown in Figure 1C. This SLS machine modification significantly reduced the consumption of raw material for scaffold fabrication.
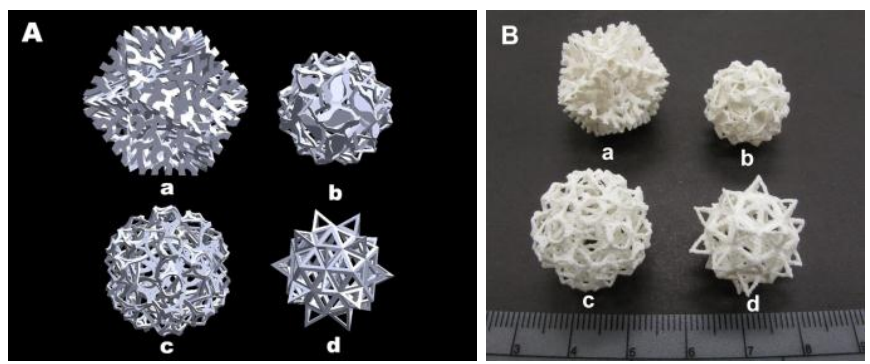

Fig. 2. Fabrication of complex porous structures via SLS: (A) four models designed by Hart for SLS [19], (a) Tangled Reindeer; (b) Salamanders; (c) Snarl and (d) elevated cosidodecahedron; (B) Ca$\mathrm{P} / \mathrm{PHBV}$ porous structures produced using the optimized values of SLS parameters.
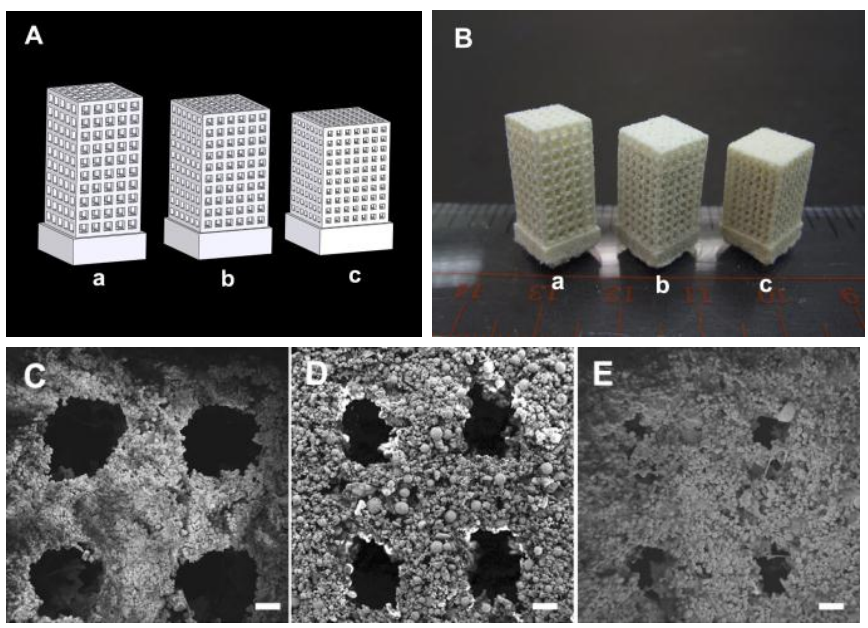

Fig. 3. Fabrication of scaffolds via SLS: (A) designed scaffold models with different pore sizes; (B) Ca-P/PHBV nanocomposite scaffolds produced, (a) Type 1, (b) Type 2, (c) Type 3; and SEM images of sintered Ca-P/PHBV nanocomposite scaffolds with different pore sizes: (C) Type 1, (D) Type 2, (E) Type 3. (Scale bar: $200 \mu \mathrm{m})$

In order to verify the optimized values of SLS parameters for Ca-P/PHBV scaffolds and investigate the fabrication of scaffolds with possible complex porous structures, Ca-P/PHBV porous structures with complex shapes and architectures were made via SLS. Using the four complex models designed by Hart for SLS (Figure 2A), four Ca-P/PHBV structures were sintered and are displayed in Figure 2B. It can be seen that provided that optimized values of SLS parameters were used, intricate porous structures with complicated shapes could be made via SLS and Ca-P/PHBV microspheres could be used for these structures. The unsintered, loose $\mathrm{Ca}-\mathrm{P} / \mathrm{PHBV}$ microspheres in the 
structures were removed easily from the structures by gentle shaking. Furthermore, the sintered structures were robust, capable of withstanding the routine handling force without any damage.

\subsection{Scaffold characteristics and selection of pore size}

Three similar scaffold models with different pore size $(0.6 \mathrm{~mm}, 0.8 \mathrm{~mm}$ and $1.0 \mathrm{~mm})$ were designed (Figure $3 \mathrm{~A}$ and Table 1). All models included one solid base and a ten-layer scaffold in the z-direction. In order to keep the cross-section areas that were occupied by scaffold struts comparable among the three types of scaffolds, there were different scaffold layers in the $\mathrm{x}$ direction and y-direction (5 layers for Type 1, 6 layers for Type 2 and 7 layers for Type 3). Ca-P/PHBV scaffolds were successfully sintered based on these three models and are shown in Figure 3B. The measured porosity values of Type 1 and Type 2 scaffolds produced were $80.7 \pm 0.7 \%$ and $62.6 \pm 1.2 \%$, respectively, which were larger that those of theoretical values listed in Table 1 (67.9\% for Type 1 and $52.7 \%$ for Type 2 ). However, the porosity of Type 3 scaffolds produced was $36.9 \pm 1.4 \%$, which was smaller than that of the theoretical value (38.0\%).

The typical layer morphology of SLS-formed CaP/PHBV nanocomposite scaffolds, which had the same designed strut size $(0.5 \mathrm{~mm})$ but different designed pore sizes, is shown in Figure $3 \mathrm{C}$ to $3 \mathrm{E}$. For sintered Type 1 and Type 2 scaffolds (with the designed pore size of $1.0 \mathrm{~mm}$ and $0.8 \mathrm{~mm}$, respectively), the pores were clearly identified and the pore size and strut size were comparable to those of the design. Similarly, both macropores of the design and micropores existing between fully sintered microspheres were observed. However, for Type 3 scaffolds (with the designed pore size of $0.6 \mathrm{~mm}$ ), the observed pore size was much smaller than the design. As expected, the mechanical properties increased with decreasing pore size. The compressive strength and modulus of Type 1 scaffolds were $0.24 \pm 0.02 \mathrm{MPa}$ and $3.96 \pm 0.64 \mathrm{MPa}$, respectively. With decreasing pore size, the compressive strength of Type 2 and Type 3 scaffolds increased to $0.55 \pm 0.09 \mathrm{MPa}$ and $0.89 \pm 0.22 \mathrm{MPa}$, respectively, with the modulus reaching $6.41 \pm 1.77$ $\mathrm{MPa}$ and $13.68 \pm 1.02 \mathrm{MPa}$, respectively. The increase of mechanical properties for Type 3 scaffolds was due not only to the decrease in pore size but also to the entrapped microspheres. Considering the requirement of pore size for bone tissue engineering, quality of sintered scaffolds and their mechanical properties, CaP/PHBV nanocomposite scaffolds with the designed pore size of $0.8 \mathrm{~mm}$ were fabricated and used in subsequent in vitro and in vivo studies.

\subsection{Surface modification of Ca-P/PHBV scaffolds}

A 3D periodic porous scaffold model was designed for the surface modification study and Ca-P/PHBV nanocomposite scaffolds were fabricated via SLS (Figure 4A). (The rod-shaped scaffold models consisted of a repeating array of struts with diameter of $1.0 \mathrm{~mm}$ in the three principal directions and the distance between each strut was set at $1.8 \mathrm{~mm}$.) Under SEM at high magnifications, both necking of microspheres and intact microspheres without fusion were observed (Figure 4B). The porosity of sintered scaffolds was measured to be $61.8 \pm 1.2 \%$, which was also higher than the theoretic porosity value $(53.5 \%)$.

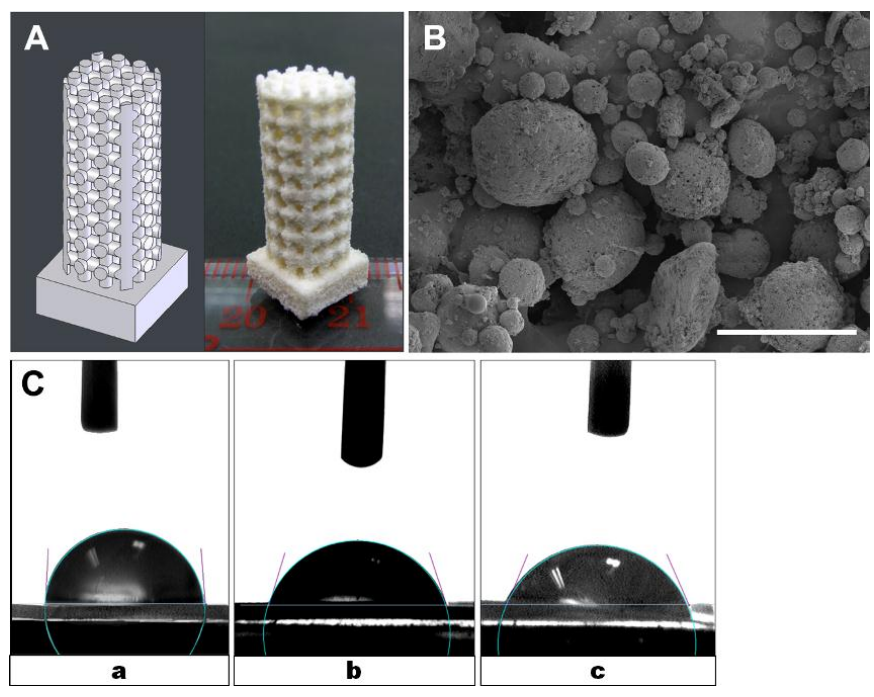

Fig. 4. Surface modification of scaffolds: (A) rod-shaped CaP/PHBV nanocomposite scaffolds for surface modification, design model (left) and sintered scaffold (right); (B) SEM image of the strut surface of a sintered Ca-P/PHBV nanocomposite scaffold; (C) water contact angle images for (a) PHBV film; (b) gelatin entrapped PHBV film; and (c) gelatin entrapped, heparin immobilized PHBV film.

For surface modification, the entrapment of gelatin was first conducted, which involved the use of a 
miscible mixture of solvent and non-solvent for the PHBV matrix. During the entrapment process, the PHBV matrix swelled but did not dissolve in the gelatin solution. The gelatin molecules then diffused onto the swollen surface of PHBV and were entangled with PHBV molecules on the surface. After the scaffold was removed from gelatin solution and immersed in water, which is a non-solvent for the polymer, the surface rapidly shrank and gelatin molecules on the polymer surface were entrapped and immobilized. This method was also employed by others [20]. The amount of entrapped gelatin was $1955.8 \pm 62.1 \mu \mathrm{g} / \mathrm{scaffold}$, as measured by the BCA kit. After gelatin entrapment, heparin was covalently coupled to amine groups of entrapped gelatin molecules. Using the Toluidine blue method, which is a facile and qualitative way to verify and determine the presence of conjugated heparin [21], a uniform blue colour caused by toluidine blue staining was observed on the scaffold surface and the amount of immobilized heparin was determined to be $41.78 \pm 0.39 \mu \mathrm{g}$ heparin per scaffold. Pore surfaces inside the scaffolds were believed to be uniformly modified by gelatin entrapment and heparin immobilization.

The surface modification did not affect the surface morphology of sintered Ca-P/PHBV nanocomposite scaffolds. Solvent-cast PHBV films were used instead of scaffolds for water contact angle measurement due to the high porosity of scaffolds. As shown in Figure $4 \mathrm{C}$, the water contact angle of PHBV films decreased drastically after surface modification. It was changed from $93.61 \pm 1.85^{\circ}$ prior to surface modification to $75.12 \pm 3.79^{\circ}$ after gelatin entrapment. Therefore, the gelatin entrapment could improve the hydrophilicity of PHBV (and hence the scaffolds). After immobilization of heparin, the water contact angle was further reduced to $72.06 \pm 0.89^{\circ}$.

\subsection{In vitro biological evaluation}

Figure 5 shows representative cell morphology on $\mathrm{Ca}$ $\mathrm{P} / \mathrm{PHBV}$ nanocomposite scaffolds with and without surface modification. Obviously, hUCMSCs became confluent on the sintered scaffolds after 21 day culture and covered all scaffold surfaces. From the high magnification examination of cell-scaffold construct surfaces, it was observed that hUCMSCs spread well and anchored themselves onto the nanocomposite microspheres of scaffold struts (Figure 5B and 5D).

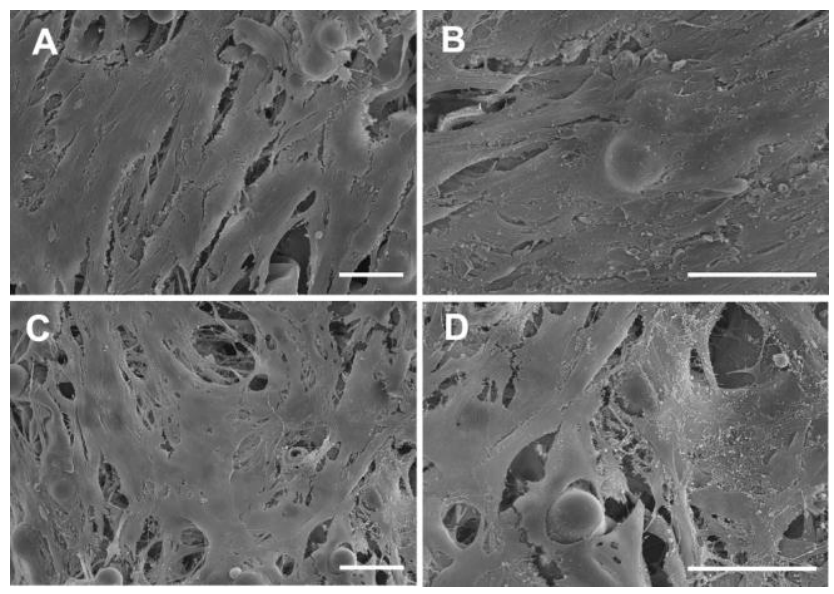

Fig. 5. Morphology of hUCMSCs cultured on Ca-P/PHBV nanocomposite scaffolds with or without surface modification for 21 days: (A, B) scaffold without surface modification; (C, D) scaffold with surface modification. (A, C: general view; B, D: close view. Scale bar: $50 \mu \mathrm{m})$

The proliferation of hUCMSCs cultured on $\mathrm{Ca}$ P/PHBV scaffolds with and without surface modification was quantified using MTT assay and the results are displayed in Figure 6A. The absorbance for all samples increased from day 1 to day 7 , indicating the trend of cell proliferation on all substrates. In the early cell culture period, Ca-P/PHBV nanocompoiste scaffolds with surface modification showed significantly higher cell proliferation $(p<0.01$ on day 1 and $p<0.05$ on day 4) than the scaffolds without surface modification. At day 7 of cell culture, no significant difference in cell proliferation was observed between scaffolds with and without surface modification.

After cell culture in the osteogenic medium, the ALP activity of hUCMSCs seeded on the four types of scaffolds, namely, Ca-P/PHBV scaffolds, surface modified Ca-P/PHBV scaffolds, Ca-P/PHBV scaffolds with rhBMP-2, and surface modified Ca-P/PHBV scaffolds with rhBMP-2, were analyzed and the results are presented in Figure 6B. For all types of scaffolds, in general, the ALP activity increased between day 7 and 14 and reached the maximum at day 14 , indicating osteogenic differentiation. Cells on surface modified scaffolds loaded with rhBMP-2 exhibited the highes enzyme activity. They showed significantly higher ALP expression than those seeded on scaffolds with simple adsorption of rhBMP-2 on day 7 and day 21 
( $p<0.05$ on day 7 and $p<0.01$ on day 21 ). Without the presence of rhBMP-2, no significant difference in ALP was observed between the scaffolds with and without surface modification. The ALP activity on scaffold samples, as well as tissue culture plates (the positive control), decreased on day 21, which is because ALP is an early marker for osteogenic differentiation and its activity usually peaks at the early stage of differentiation
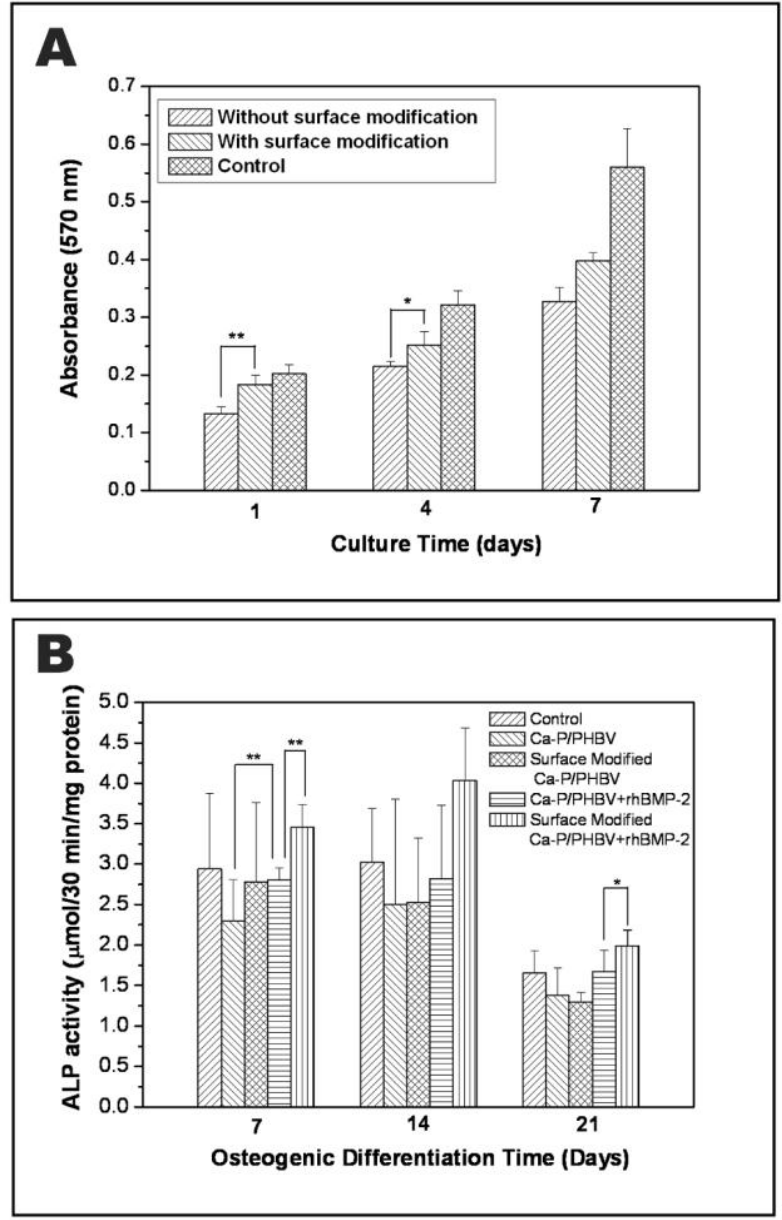

Fig. 6. In vitro cell culture study: (A) MTT assay results for cell proliferation of hUCMSCs on the Ca-P/PHBV nanocomposite scaffolds with or without surface modification; (B) ALP activity of hUCMSCs seeded on different scaffolds and tissue culture plate (the control) for different culture times. $\left({ }^{*} p<0.05\right.$; $* * p<0.01)$

\subsection{Ectopic bone formation}

After 6 week and 12 week implantation, nanocomposite scaffolds implanted in the muscles of rabbits were collected and are shown in Figure 7C and 7D. Six weeks after implantation, no obvious bone formation was observed for both simple Ca-P/PHBV scaffold and Ca-P/PHBV scaffold loaded with rhBMP2 (Figure 7C). Twelve weeks after implantation, only soft tissue was observed around Ca-P/PHBV scaffold without rhBMP-2. In contrast, the Ca-P/PHBV scaffold loaded with rhBMP-2 was surrounded by bony tissue (Figure 7D).
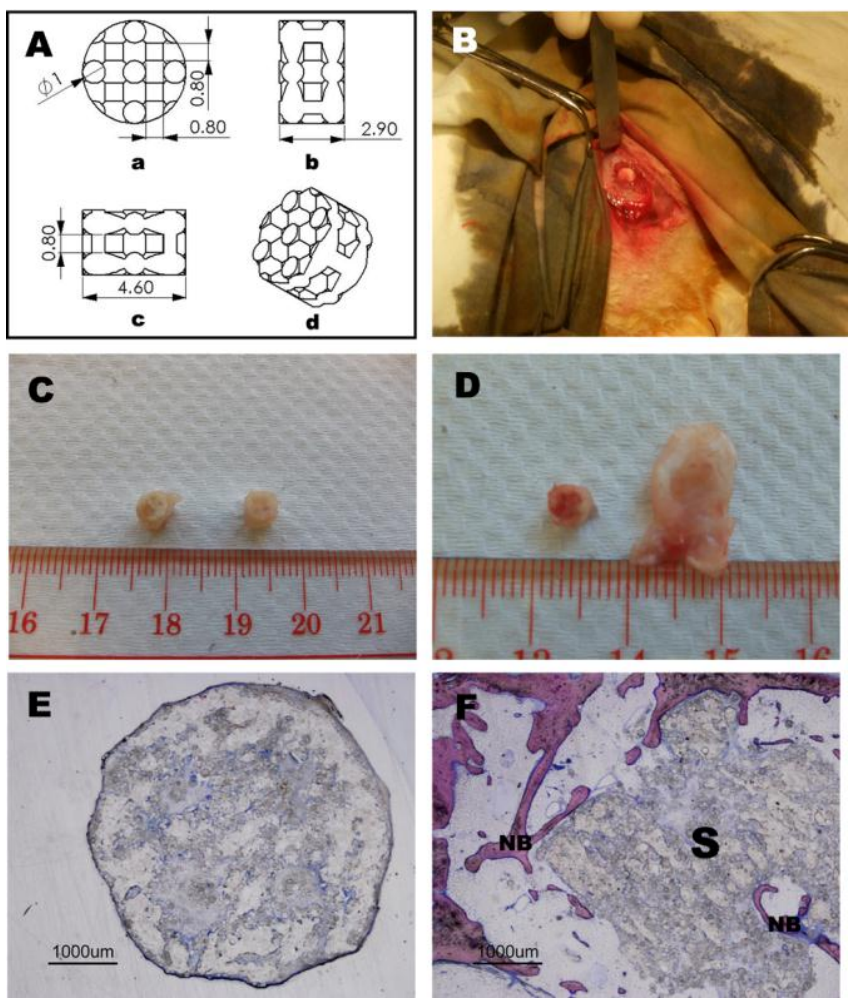

Fig. 7. In vivo study of Ca-P/PHBV nanocomposite scaffolds: A: Scaffold model for the in vivo study (dimensions in $\mathrm{mm}$ ), (a) top view, (b, c) side views, (d) trimetric view; B: Implantation of a scaffold in the muscle pocket in a rabbit; C, D: Harvested samples after intramuscular implantation in rabbits for different times (Left $\mathrm{Ca}$-P/PHBV nanocomposite scaffold; right: surface modified CaP/PHBV nanocomposite scaffold loaded with rhBMP-2), (C) 6 weeks, (D) 12 weeks; E, F: histological images of different scaffolds after 12 week intramuscular implantation in rabbits, (E) Ca-P/PHBV nanocomposite scaffold, (F) surface modified CaP/PHBV nanocomposite scaffold loaded with rhBMP-2.

The representative histological images of tissuescaffold samples are shown in Figure 7E and 7F. These images were obtained from samples after 12 week implantation and were stained with giemsaeosin. (Scaffolds after 6 week implantation were encapsulated by the fibrous connective tissue rather than bone and the data are not shown in this paper.) At 12 weeks, the formation of new bone, stained as the pink color in Figure 7F, was observed in the $\mathrm{Ca}$ P/PHBV scaffold loaded with rhBMP-2, whereas, as 
expected, the simple Ca-P/PHBV scaffold did not induce any bone formation. The bony tissue also grew into the pores of rhBMP-2 loaded scaffolds.

\section{Discussion}

The current investigation firstly demonstrated the success of the strategies adopted, viz., forming the $\mathrm{Ca}$ $\mathrm{P} / \mathrm{PHBV}$ nanocomposite as a scaffold material for bone tissue engineering and using the SLS technique to fabricate 3D nanocomposite scaffolds with controlled porous structure and properties. The $\mathrm{Ca}$ P/PHBV nanocomopsite consists of a PHBV matrix, which is a well-established biodegradable polymer for medical applications, and nano-sized Ca-P particles. Scaffolds made of this composite mimic the structure and functions of bone ECM to support expanded cells, which will secrete their own ECMs, replacing the biodegradable scaffolds with new bone tissue. Another advantage of the current processing route, as compared to the dry-blending method adopted by some researchers in preparing SLS raw materials, is that the bioceramic nanoparticles can be homogeneously distributed in the polymer matrix of composite scaffolds. In addition, using this processing route, some drugs or even biomolecules can be incorporated into the microspheres (and hence the scaffolds after SLS) in order to expand the functions of the scaffolds. Using various simple or complex models (Figure 2A, 3A, 4A and 7A), robust $\mathrm{Ca}-\mathrm{P} / \mathrm{PHBV}$ nanocomposite scaffolds and porous structures were successfully produced, which had comparable features as the design models. The current investigation has thus demonstrated that according to specific requirements and designs, osteoconductive bone tissue engineering scaffolds can be made via SLS using the Ca-P/PHBV nanocomposite microspheres.

During bone regeneration, characteristics of pores in scaffolds are of significant importance because the pores allow migration and proliferation of osteoblasts and mesenchymal cells, as well as vascularization [22]. Conflicting data exist in the open literature with regard to the effects of pore size, size distribution and porosity on osteogenesis and vascularization. Usually, pore sizes in the range of 200 to $900 \mu \mathrm{m}$ are considered to be most suitable for synthetic bone grafts designed to mimic the architecture of trabecular bone [23]. Furthermore, the pore size in scaffolds may also influence the type of osteogenesis. Several studies on ectopic bone formation using hydroxyapatite reported that smaller pore sizes $(90-120 \mu \mathrm{m})$ resulted in chondrogenesis before osteogenesis whereas larger pore sizes $(350 \mu \mathrm{m})$ resulted in direct osteogenesis with enhanced vascularization [24, 25]. From the mechanical point of view, structures with smaller pore size provide greater mechanical stability in a complex mechanical environment. Therefore, a balance is needed between a large pore size that allows rapid osteointegration and a small pore size that provides the ability to bear mechanical loads. In the current investigation, a pore size was chosen on the basis of bone tissue engineering requirements, resolution of the SLS equipment and sintered products. With a large design pore size $(0.8 \mathrm{~mm}$ or $1.0 \mathrm{~mm})$, sintered scaffolds (Type 1 and Type 2 scaffolds) had a larger porosity than the design value but possessed a lower mechanical strength. In contrast, the porosity of sintered Type 3 scaffolds was smaller than that of the theoretical value. The laser spot size in the Sinterstation ${ }^{\circledR} 2000$ system, which is determining factor for the resolution of sintered products, is about $457 \mu \mathrm{m}$, which is closer to the design pore size of Type 3 scaffolds $(600 \mu \mathrm{m})$. Therefore, the pore size in Type 3 scaffolds was not as accurate as those in Type 1 and Type 2 scaffolds. In addition, the "growth effect", which is a common phenomenon in SLS processes, also significantly affects the feature dimensions in sintered scaffolds. This phenomenon is usually observed in the sintered products with small features or small pores due to the penetration of the laser energy beyond the theoretical scan area. The growth effect had decreased the pore size of Type 3 scaffolds and thus increased the strut size, resulting in a lower porosity than the theoretical value. Normally, the entrapped unsintered microspheres were easily removed from the Type 1 and Type 2 scaffolds by manual shaking. However, for Type 3 scaffolds, the entrapped microspheres could not be completely removed even with the help of a compressed gas jet. Therefore, the design pore size of $0.8 \mathrm{~mm}$ for Type 2 scaffolds was considered as suitable pore size in the current investigation on the basis of the pore size requirement for bone regeneration, quality of sintered scaffolds and their mechanical properties. By controlling the architecture of the scaffolds (pore size, 
interconnectivity, geometry, etc.), the physical, mechanical and biological properties of scaffolds such porosity and density, strength, degradation and cellular response could be controlled.

In order to improve the bioactivity of sintered scaffolds and thus promote bone regeneration, growth factors can be incorporated in bone tissue engineering scaffolds. For achieving a better control of the growth factor release behavior, in the current investigation, heparin was immobilized on the surface of gelatin entrapped Ca-P/PHBV nanocomposite scaffolds. Based on the carbodiimide chemistry, the entrapped gelatin provided amino group for the conjugation of heparin. Heparin, a sulfated polysaccharide belonging to the glycosaminoglycans family, is known to have the binding affinity with a number of growth factors and is thus capable of blocking the degradation of the growth factors and prolonging their release time [26, 27]. The surface modification by the entrapment of gelatin and immobilization of heparin greatly decreased the water contact angle of solvent-cast PHBV films, indicating the large improvement of wettability of surface modified Ca-P/PHBV nanocomposite scaffolds. But the morphology and mechanical properties of $\mathrm{Ca}-\mathrm{P} / \mathrm{PHBV}$ nanocomposite scaffolds were not altered by this surface modification.

hUCMSCs are a promising cell source for bone tissue regeneration due to their inexhaustible supply, multilineage differentiation capacity and noninvasive harvest procedure. The behavior and fate of stem cells are usually regulated by their in vivo microenvironment, known as stem cell niche [28]. Key niche components and interactions include growth factors, cell-cell contacts, and cell-ECM adhesions [29]. The role of tissue engineering scaffolds is generally to biomimic the natural ECM and to emulate stem cell niche in combination with growth factors and other signaling. In the current investigation, 3D CaP/PHBV nanocomposite scaffolds with surface modification, i.e., gelatin entrapment and heparin immobilization, and further loading of rhBMP-2 were used to investigate the osteogenic differentiation of hUCMSCs. The improvement of wettability resulting from the surface modification facilitated the adhesion of hUCMSCs at the early stage of cell culture (in culture before 7 days) when cells made contact with the scaffolds. However, no significant difference was observed in ALP activity in the osteogenic differentiation of hUCMSCs cultured on Ca-P/PHBV nanocomposite scaffolds with and without surface modification. The cells cultured on Ca-P/PHBV nanocomposite scaffolds with and without surface modification became confluent and showed normal phenotype after 21 days in the osteogenic medium. The main purpose of heparin immobilization on scaffolds was to provide specific affinity between heparin immobilized scaffolds and growth factors and to control the release profile of bound growth factors in a sustained manner so as to improve angiogenesis or osteogenesis [30, 31]. The ALP activity assay results showed that the ALP level was significantly upregulated on surface modified Ca-P/PHBV scaffolds loaded with rhBMP-2 as compared to that on scaffolds with the simple adsorption of rhBMP-2. This clearly indicates that the osteogenic differentiation of hUCMSCs on scaffolds loaded with rhBMP-2 was affected by the way rhBMP-2 was incorporated and that it was important to provide immobilized heparin.

Ectopic bone formation by surface modified $\mathrm{Ca}-$ P/PHBV nanocomposite scaffolds was studied in the current investigation using a rabbit model. For intramuscular implantation, the Ca-P/PHBV nanocomposite scaffolds without rhBMP-2 did not induce any bone formation after implantation for up to 12 weeks. In high contrast, surface modified scaffolds loaded with rhBMP-2 stimulated ectopic bone formation after 12 week implantation, indicating the positive effects of rhBMP-2 release on homologous bone formation. The moderate foreign body reaction and the absence of necrosis at the implantation site indicated that there were no in vivo toxic effects by the Ca-P/PHBV nanocomposite scaffolds. The in vivo results suggested that the surface modified $\mathrm{Ca}$ P/PHBV nanocomposite scaffolds loaded with rhBMP-2 possessed osteoinductivity and therefore could promote bone tissue formation in both nonosseous and osseous sites in the body.

\section{Conclusions}

It is feasible to combine the SLS technique, nanocomposite approach and sustained release of growth factor to form high-performance bone engineering scaffolds. Scaffolds and porous structures of complex shapes and architecture could be fabricated 
via SLS. For Ca-P/PHBV nanocomposite scaffolds, with decreasing pore size, the porosity of scaffolds decreased and the mechanical properties increased. Nanocomposite scaffolds with the design pore size of $0.8 \mathrm{~mm}$ were considered to be suitable for bone tissue engineering. The surface modification improved the wettability of scaffolds and provided binding sites for the growth factor rhBMP-2. The ALP activity of hUCMSCs on surface modified scaffolds loaded with rhBMP-2 was significantly higher than that of the cells on scaffolds with simple adsorption of rhBMP-2 during the 21 day culture period. In in vivo experiments using a rabbit model, ectopic bone formation by surface modified Ca-P/PHBV scaffolds loaded with rhBMP-2 occurred after 12 week intramuscular implantation. Integrating SLS for scaffold fabrication, osteoconductive nanocomposite as scaffold material and controlled growth factor delivery provides a very effective strategy for bone tissue engineering.

\section{Acknowledgments}

B. Duan thanks The University of Hong Kong (HKU) for making the University Scholarship Award to him during his $\mathrm{PhD}$ study at HKU. This work was supported by GRF grants (HKU 7182/05E and HKU 7181/09E) from the Research Grants Council of Hong Kong and also by a research grant from HKU.

\section{References}

1. R. Skalak and C. F. Fox, Tissue Engineering, (Alan R.Liss Inc., New York, 1988).

2. A. J. Salgado, O. P. Coutinho and R. L. Reis, Macromolecular Bioscience 4, 743 (2004).

3. J. M. Kanczler and R. O. C. Oreffo, European Cells \& Materials 15, 100 (2008).

4. M. Wang, Biomaterials 24, 2133 (2003).

5. J. R. Porter, T. T. Ruckh and K. C. Popat, Biotechnology Progress 25, 1539 (2009).

6. J. A. Kanczler, P. J. Ginty, J. J. A. Barry, N. M. P. Clarke, S. M. Howdle, K. M. Shakesheff and R. O. C. Oreffo, Biomaterials 29, 1892 (2008).

7. B. Li, T. Yoshii, A. E. Hafeman, J. S. Nyman, J. C. Wenke and S. A. Guelcher, Biomateirals 30, 6768 (2009).

8. C. K. Chua, K. F. Leong and C. S. Lim, Rapid prototyping: principles and applications. (World Scientific, Singapore, 2010).

9. K. F. Leong, C. M. Cheah and C. K. Chua, Biomaterials 24, 2363 (2003).
10. E. Berry, J. M. Brown, M. Connell, C. M. Craven, N. D. Efford, A. Radjenovic and M. A. Smith, Medical Engineering \& Physics 19, 90 (1997).

11. R. Petzold, H. F. Zeilhofer, W. A. Kalender, Computerized Medical Imaging and Graphics 23, 277 (1999).

12. L. Hao, M. M. Savalani, Y. Zhang, K. E. Tanner, R. J. Heath and R. A. Harris, Proceedings of the Royal Society A: Mathematical Physical and Engineering Sciences 463, 1857 (2007)

13. M. A. Lopez-Heredia, J. Sohier, C. Gaillard, S. Quillard, M. Dorget and P. Layrolle, Biomaterials 29, 2608 (2008).

14. T. B. F. Woodfield, M. Guggenheim, B. von Rechenberg, J. Riesle, C. A. van Blitterswijk and V. Wedler, Cell Proliferation 42, 485 (2009).

15. N. A. Silva, A. J. Salgado, R. A. Sousa, J. T. Oliveira, A. J. Pedro, H. Leite-Almeida, R. Cerqueira, A. Almeida, F. Mastronardi, J. F. Mano, N. M. Neves, N. Sousa and R. L. Reis, Tissue Engineering Part A 16, 45 (2010).

16. B. Duan, M. Wang, W. Y. Zhou and W. L. Cheung, Applied Surface Science 255, 529 (2008).

17. B. Duan, M. Wang, W. Y. Zhou, W. L. Cheung, Z. Y. Li and W. W. Lu, Acta Biomaterialia 6, 4495 (2010).

18. W. Y. Zhou, S. H. Lee, M. Wang, W. L. Cheung and W. Y. Ip, Journal of Materials Science-Materials in Medicine 19, 2535 (2008)

19. Hart, G. W. "http://www.georgehart.com/rp/rp.html". (accessed on December 1st 2009 ), the State University of New York in Stony Brook, New York, USA (2009).

20. X. H. Liu, Y. J. Won and P. X. Ma, Journal of Biomedical Materials Research Part A 74A 84 (2005).

21. J. S. Son, K. Park, S. Oh, J. J. Kim and D. K. Han, Tissue Engineering and Regenerative Medicine 5, 528 (2008).

22. S. Y. Kim and G. I. Im, Tissue Engineering and Regenerative Medicine 6, 1391 (2009).

23. T. Mygind, M. Stiehler, A. Baatrup, H. S. Li, X. N. Zou, A. Flyvbjerg, M. Kassem and C. Bunger, Biomaterials 28, 1036 (2007).

24. Y. Kuboki, Q. M. Jin and H. Takita, Journal of Bone and Joint Surgery-American Volume 83A, S105 (2001).

25. C. E. P. Aronin, , K. W. Sadik, , A. L. Lay, D. B. Rion, S. S. Tholpady, R. C. Ogle, E. A. Botchwey, Journal of Biomedical Materials Research Part A 89A, 632 (2009)

26. I. Capila and R. J. Linhardt, Angewandte ChmieInternational Edition 41, 391 (2002).

27. X. Y. Jiao, P. C. Billings, M. P. O'Connell, F. S. Kaplan, E. M. Shore and D. L. Glaser, Journal of Biological Chemistry 282, 1080 (2007).

28. T. Vazin and D. V. Schaffer, Trends in Biotechnology. 28, 117 (2010).

29. D. E. Discher, D. J. Mooney, P. W. Zandstra, Science 324, 1673 (2009).

30. L. Chen, Z. Q. He, B. Chen, M. J. Yang, Y. N. Zhao, W. J. Sun, Z. F. Xiao, J. Zhang and J. W. Dai, Journal 
of Materials Science-Materials in Medicine 21, 309 (2010).

31. J. A, Beamish, L. C. Geyer, N. A. Haq-Siddiqi, K. Kottke-Marchant and R. E. Marchant, Biomaterials 30, 6286 (2009). 